\title{
Autobiographical memory in multiple sclerosis patients: Assessment and cognitive facilitation
}

\author{
${ }^{1}$ A. Ernst ${ }^{\text {a }}$, F. Blanc ${ }^{\text {a,c }}$, V. Voltzenlogel ${ }^{\text {b }}$, J. de Seze ${ }^{\text {a,c }}$, B. Chauvin ${ }^{\text {d } ~ \& ~ L . ~ M a n n i n g ~ a ~}$ \\ ${ }^{a}$ Imaging and Cognitive Neurosciences Laboratory, University of Strasbourg, Strasbourg, France \\ ${ }^{b}$ Octagon Laboratory, CERPP and Toulouse Le Mirail University, Toulouse, France \\ ${ }^{c}$ Neurology Unit, Strasbourg University Hospitals, Strasbourg, France \\ ${ }^{d}$ Psychology of Cognition Laboratory, University of Strasbourg, Strasbourg, France
}

\begin{abstract}
The multifocal nature of lesions in multiple sclerosis hints at the occurrence of autobiographical memory (AbM) impairment. However, the dearth of studies on $\mathrm{AbM}$ in multiple sclerosis is noticeable, notwithstanding the importance of $\mathrm{AbM}$ in everyday life. In the first section of this study, 25 multiple sclerosis patients and 35 controls underwent a detailed episodic AbM assessment. Results obtained by means of ANOVA suggested an AbM retrieval deficit in every patient. That pattern of performance paved the way for the second section of the study, in which we followed up 10 out of the 25 patients. Our objective was to assess the effectiveness of a cognitive facilitation programme designed to alleviate AbM retrieval deficits, based on the key role of mental visual imagery on AbM. Statistical group analyses by means of ANOVA and individual analyses using the $\chi^{2}$ test showed significant differences in AbM test results, in post-facilitation relative to pre-facilitation training, in all 10 patients. Moreover, the patients' comments showed that the positive effects were transferred in their daily life functioning. We would like to suggest that the facilitation programme efficiently enhanced the process of selfcentred mental visual imagery, which might have compensated for poor retrieval of personal memories by providing better access to visual details and detailed visual scenes of personal recollections.
\end{abstract}

Keywords : Multiple sclerosis ; Autobiographical memory ; Cognitive facilitation ; Mental visual imagery.

\section{INTRODUCTION}

Multiple sclerosis (MS) is an immune inflammatory pathology characterised by the multifocal nature of neurological lesions and the diversity of its neu-ropathological processes (i.e., demyelination, white and grey matter injuries, atrophy; Trapp \& Nave, 2008), which results in a wide variety of symptoms.

With regard to cognition in MS, Rao, Leo, Bernardin, and Unverzagt (1991) demonstrated cognitive impairments in $30-70 \%$ of MS patients. The cognitive domains that are frequently reported as being affected include attention, information processing speed, executive functions and, particularly, anterograde memory, which seems to be the most frequently impaired function (40-60\% of MS patients; Calabrese, 2006; Rogers \& Panegyres, 2007). Although there is no consensus about the memory process that would be predominantly altered in MS, according to several authors, the retrieval process might be more sensitive to MS effects than the encoding or consolidation processes (Calabrese, 2006; Drew, Tippett, Starkey, \& Isler, 2008; Rogers \& Panegyres, 2007; Sartori \& Edan, 2006; Zakzanis, 2000).

More relevant for the present study, is the relationship between remote memory and MS. Two dimensions of personal remote memory seem particularly important in the present context: its influence in everyday life and its neuroanatomical characteristics. We briefly develop these issues before considering personal remote memory in MS patients. Personal remote memory encompasses semantic and episodic components. The former corresponds to factual personal knowledge (personal semantics) with no sense of reliving the event during its recollection

\footnotetext{
${ }^{1}$ We are grateful to Michel Clanet and David Brassat for patient referral and to Maria-José Quiñonez, Fanny Durozier, Léna Vanoli, Clémence Kuhn and Mathieu Leininger for their contribution to testing patients and healthy subjects. We thank Anne Botzung and Nathalie Heider for their useful comments on the manuscript. This study is part of a larger research project financed by the Fondation pour la Recherche sur la Sclérose en Plaques (ARSEP), Ile de France, to LM.
} 
(Tulving, Schacter, McLachlan, \& Moscovitch, 1988). The second component is typically represented by autobiographical memory $(\mathrm{AbM})$ which refers to the capacity of reliving detailed events, within their specific spatio-temporal context, as they are remembered (Tulving, 1985).

Autobiographical memory is central in everyday normal functioning. Indeed, since it contributes to the construction and continuity of the self, it plays at least two social roles (the nurturing of existing relationships and the development of new ones) and involves a directive function, where the past serves as a basis for guiding present and future behaviours (Rasmussen \& Habermas, 2011).

From a neuroanatomical perspective, AbM recruits a widespread cerebral network, predominantly in the left and medial regions, including as core structures the prefrontal cortex, medial and lateral temporal cortices, parietooccipital regions, temporoparietal junctions and cerebellum (see Svoboda, McKinnon, \& Levine, 2006, for a review). As a consequence of this extensive cerebral recruitment, AbM is highly vulnerable to brain damage (Addis, Sacchetti, Ally, Budson, \& Schacter, 2009; Matuszewski et al., 2006; Miller et al., 2001; Voltzenlogel et al., 2006). Conversely, personal semantic memory seems more resistant to brain damage (Moscovitch, Rosenbaum, Gilboa, Addis, \& Westmacott, 2005) and relies mostly on the lateral temporal cortex (Svoboda et al., 2006). Bearing in mind the neuroanatomical observations in relation to AbM as well as the fact that MS is characterised by multifocal cerebral lesions, we surmised that AbM might be particularly prone to be impaired in MS. This suggestion is supported by the following clinical signs. MS patients happen to mention difficulties in social situations, in which AbM partaking is ubiquitous (Suddendorf \& Corballis, 1997). Moreover, in everyday clinical work, it is noticeable that patients experience difficulties in reporting precise personal past events.

However, with regard to the literature on remote memory in MS patients, to the best of our knowledge, only two studies have investigated this subject: Paul, Blanco, Hames, and Beatty (1997) and Kenealy, Beaumont, Lintern, and Murrel (2002). It appears that several limitations (see the Discussion section) restrict the conclusions that can be drawn from these two studies.

As mentioned above, when considering anterograde memory studies in MS, the memory process that seems to be most impaired in MS is the retrieval process. This observation is relevant also for remote memory since the retrieval process is crucial in $\mathrm{AbM}$ and several authors have pointed out the central role of prefrontal regions during the retrieval of personal recollections in healthy subjects (Botzung, Denkova, Ciuciu, Scheiber \& Manning, 2008; Manenti, Cotelli, Calabria, Maioli \& Minussi, 2010), as well as neurological patients (Matuszewski et al., 2006; Montoya et al., 2006; Smith, Souchay, \& Conway, 2009; Zarei, Pourtemad, Bak, \& Hodges, 2010). Bearing these results in mind, the greater occurrence of frontal cortex lesions in MS (Arnett, 2003; Bendfeldt et al., 2009; Benedict et al., 2002; Sperling et al., 2001) and the studies that conclude there is frontal lobe dysfunction in MS patients (Drew et al., 2008; Santiago, Guardia, Casado, Carmona, \& Arbizu, 2007) suggest a predominant strategy deficit in MS patients, who complain, in everyday clinics, of difficulties in AbM. We have investigated formally this issue, hypothesising that the AbM deficit in MS could rather be due to a failure to access otherwise preserved recollection representations (Rapp \& Camarazza, 1993; Warrington \& Shallice, 1979). Moreover, given the importance of AbM in everyday life, pending the confirmation of our hypothesis, we endeavoured to develop and implement a tailor-made facilitation programme conceived to back up access to past memories.

Turning presently to cognitive rehabilitation in MS (see O'Brien, Chiaravalloti, Goverover, \& DeLuca, 2008, for a review), anterograde memory has been targeted in a number of studies and several rehabilitation methods have been probed that aimed at improving learning abilities in MS patients (Allen, Goldstein, Heyman, \& Rondinelli, 1998; Basso, Lowery, Ghormley, Combs, \& Johnson, 2006; Chiaravalloti \& DeLuca, 2002; Chiaravalloti, Demaree, Gaudino, \& DeLuca, 2003; Chiaravalloti, DeLuca, Moore, \& Ricker, 2005; Goveover, Hillary, Chiaravalloti, Arango-Lasprilla, \& DeLuca, 2009; Hildebrant et al., 2007; Mendozzi et al., 1998; Solari et al., 2004). Among them, the use of mental visual imagery (MVI) techniques lead to positive outcomes in relapsingremitting MS patients with moderate to severe memory impairment (Chiaravalloti et al., 2005). However, an overview of anterograde memory rehabilitation in MS shows no general agreement either about the comparison of pre- and post-cognitive rehabilitation results or about the transfer of benefits in everyday life. As regards rehabilitation, both measures are important, particularly the latter which is the core of "successful cognitive rehabilitation" (Wilson, 1987, 2008).

MVI - i.e., seeing with the mind's eye - is critical for the retrieval of episodic memories and vividness of remembering (Greenberg \& Rubin, 2003; Huijbers, Pennartz, Rubin, \& Daselaar, 2011; Moulton \& Kosslyn, 2009). More to the point, MVI is involved in the reconstructive process of past events and allows, most particularly, the cueing of visual information, and also information relating to other sensory modalities, about the 
event (Huijbers et al., 2011; Rubin, 2005).

The importance of MVI in AbM is supported both by case reports of autobiographical amnesic patients presenting, primarily, an MVI deficit (Greenberg, Eacott, Brechin, \& Rubin, 2005; Rubin \& Greenberg, 1998), and neuroimaging studies showing that imagination of fictitious scenes and AbM are sustained by overlapping cerebral network activations (Hassabis, Kumaran, \& Maguire, 2007; Huijbers et al., 2011). On this basis, the use of MVI in the context of the present study was promising, all the more so that MVI had already been used as a rehabilitation technique in MS (Chiaravalloti et al., 2005).

In the first part of the present work we report our investigations about AbM impairment in MS using a stringent $\mathrm{AbM}$ test. We then analyse the relationships between AbM deficits and other cognitive functions to understand the processes underlying the deficit, namely, the associations or otherwise with anterograde memory and executive function performance. In the second section of the study, we present an MVI-based cognitive facilitation programme for MS patients presenting with a selective AbM retrieval deficit. Our aim was to probe the programme under the hypothesis that the number of richly detailed episodic events evoked by the patients could be increased by reinforcing the cueing role of MVI, and by the same token, improve their AbM performance.

\section{METHODS}

\section{Subjects}

We examined 25 patients with definite MS according to the McDonald criteria (McDonald et al., 2001). Patients were recruited at the Neurology Units of four French hospitals (Strasbourg, Toulouse, Montbéliard, and Besançon). Inclusion criteria were the diagnosis of a relapsing-remitting disease course (RRMS), a mild functional disability corresponding to an Expanded Disability Status Scale (EDSS; Kurtzke, 1983) score $\leq 6$, an absence of major signs of depression according to the Beck Depression Inventory - Short form (BDI; score $\leq 13$; Beck \& Steer, 1993) and absence of anxiety based on the Hamilton Anxiety Scale (HAS; score $\leq 18$; Hamilton, 1959). A subgroup of 10 patients presenting with selective AbM impairment was drawn. General cognitive functioning (see Neuropsychological baseline examination section) was deemed necessary in order to probe the effects of our programme on AbM with as few confounding factors as possible.

A group of 35 healthy controls matched for gender, age and educational level was also recruited. Ten of them were assessed twice on the AbM test with at least a two month interval between the two sessions.

Exclusion criteria for all the participants included documented psychiatric illness, neurological disorder (other than MS for the patient group) and poor knowledge of French. Demographic and clinical data are summarised in Table 1. All subjects gave prior informed consent; we complied with the APA ethical standards; and the research was approved by the Centre Hospi-talier Universitaire de Strasbourg.

\section{Neuropsychological examination}

The following baseline tests were presented to the group of 25 patients. Verbal IQ was assessed with the short form (Warrington, James, \& Maciejewski, 1986) of the WAIS-R Verbal scale (Wechsler, 1989). Executive functions were assessed using the phonological and categorical fluency tests (National Hospital, London) and the Paced Auditory Serial Addition Test (PASAT; Gronwall, 1977). The Information Processing Speed test from the Adult Memory Information Processing Battery (AMIPB; Coughlan \& Hollows, 1985) was also administered. Since the experimental test assessing AbM is language-dependent, language was also examined, by means of the Déno 100 test (Kremin, 2002). Visuo-perceptual and visuo-spatial abilities were tested using the Silhouettes and Cube Analysis sub-tests from the Visual Object and Space Perception Battery (VOSP; Warrington \& James, 1991). Anterograde memory was examined using the Selective Reminding Test (SRT; Buschke \& Fuld, 1974) or the Rey Auditory Verbal Learning Test (RAVLT; Rey, 1964) and/or the Recognition Memory Test for Words (RMTW; Warrington, 1984) for the verbal modality. The Complex Figure Test from the AMIPB (Coughlan \& Hollows, 1985) was used to assess the visual modality of anterograde memory.

A few methodological disparities in the number of patients who completed the neuropsychological baseline were due to multisite recruitment (see Table 2 for the breakdown of patients who underwent each test). Data for the BDI and HAS were also unavailable for two patients but major signs of depression and/or anxiety were excluded based on clinical examination. The scores of the 10 patients included in the facilitation programme group had to be in the normal range on all tests (threshold: either $\mathrm{z}$-score -1.65 or the $5^{\text {th }}$ percentile, depending on normative 
data), except for the AbM score.

TABLE 1 General data: Mean and standard deviation for the whole group of MS patients and healthy controls assessed for AbM, and for the facilitation subgroup

\begin{tabular}{|c|c|c|c|}
\hline & MS patients & Healthy controls & Statistical analysis \\
\hline AbM assessment group & & & \\
\hline$N$ & 25 & 35 & - \\
\hline Age in years & $\begin{array}{l}42.96 S D 10.94 \\
(28-64)\end{array}$ & $\begin{array}{l}42.17 S D 9.44 \\
(24-59)\end{array}$ & $\begin{array}{l}t(58)=-0.30 ; p=.77 \\
-\end{array}$ \\
\hline Education in years & $\begin{array}{l}13.20 S D 2.84 \\
(9-20)\end{array}$ & $\begin{array}{l}12.71 S D 2.82 \\
(9-23)\end{array}$ & $t(58)=0.65 ; p=.52$ \\
\hline Sex (Ratio female/male) & $21 / 4$ & $29 / 6$ & $\chi^{2}=0.01 ; p=.91$ \\
\hline (range) & $\begin{array}{l}1.77 S D 1.72 \\
(0-5.5)\end{array}$ & - & - \\
\hline Duration of MS in years & $8.85 S D 7.59$ & - & - \\
\hline Facilitation subgroup & & & \\
\hline$N$ & 10 & 10 & - \\
\hline Age in years & $48.6 S D 11.46$ & $46.7 S D 11.9$ & $t(18)=0.33(p=.75)$ \\
\hline Education in years & $13.3 S D 2.31$ & 14.2SD 3.71 & $t(18)=-0.13(p=.9)$ \\
\hline Sex (Ratio female/male) & $9 / 1$ & $9 / 1$ & - \\
\hline EDSS & $2.4 S D 2.08$ & - & - \\
\hline Duration of MS in years & $10.6 S D 7.23$ & - & - \\
\hline
\end{tabular}

$\underline{T A B L E} 2$ Percentages of patients with deficient scores on neuropsychological baseline tests

\begin{tabular}{lcc}
\hline Test & N patients & Percentages of patients under normal range \\
\hline Verbal IQ & 25 & 0 \\
SRT/RAVLT & 15 & 46.67 \\
RMTW & 25 & 0 \\
AMIPB/Figure recall & 25 & 20 \\
Deno 100 & 15 & 4 \\
Silhouettes (VOSP) & 25 & 8 \\
Cube analysis (VOSP) & 25 & 0 \\
Phonological fluency & 25 & 4 \\
Categorical fluency & 25 & 0 \\
PASAT & 20 & 25 \\
AMIPB /Motor speed & 25 & 8 \\
AMIPB/Information processing & 25 & 20 \\
BDI & 23 & 0 \\
HAS & 23 & 0
\end{tabular}

SRT: Selective Reminding Test; RAVLT: Rey Auditory Verbal Learning Test; RMTW: the Recognition Memory Test for Words; AMIPB: the Adult Memory Information Processing Battery; VOSP: the Visual Object and Space Perception Battery; PASAT: Paced Auditory Serial Addition Test.

Autobiographical memory was assessed, in the 25 patients and 35 control subjects, to probe its two components, episodic and personal semantics, by means of the following tests.

The cue-words Modified Crovitz Test (MCT; Graham \& Hodges, 1997; using Manning's 2002 French version). For each cue-word (e.g., letter, friend, film) the participants were asked to produce four recollections, each associated with one of the four different life periods: 0-9 years, 10-19 years, 20-to-(current age - 1 year), and the past year. The presentation of words and life periods was randomised. No time limit was set to avoid the potential influence of decreased cognitive processing speed on AbM performances, and the participants were encouraged to provide as many details as possible. Memories were subsequently scored on a 5-point scale as 
follows: $0=$ absence of response; $1=$ semantic facts related to the target word; $2=$ poorly detailed generic events (i.e., overgeneral); 3 = richly detailed generic events (i.e., overgeneral); 4 = poorly detailed specific events (i.e., autobiographical); 5 = richly detailed specific events (i.e., autobiographical). Incomplete responses were probed with general prompts (e.g., "Tell me more"; "Can you think of a specific instance?"). The normality threshold for this test was $74 \%$ of the maximum total score (i.e., 88/120; unpublished data from Manning et al.).

The AMI (Kopelman, Wilson, \& Baddeley, 1989). This test tackled personal semantics over three lifetime periods - childhood, young adulthood and recent life.

MCT test. As 10 patients were to carry out the MCT a second time, after the facilitation programme, in order to assess its benefits, 10 control subjects were also administered the MCT a second time, at least 2 months later, with a view to provide evidence of or to rule out a possible learning bias.

\section{Autobiographical memory facilitation programme}

The cognitive facilitation programme was constructed to alleviate AbM retrieval difficulties by means of a memory facilitation technique based on MVI. This technique was presented to the patients as a "tool" to use in order to make it easier to access a given recollection and the details associated with it. Therefore, during the MVI training sessions, patients found it increasingly easier to pay close attention to details in the mind's eye, which was used spontaneously when evoking personal recollections. Moreover, they were encouraged to try and use this technique in their everyday life and if needed, to ask the neuropsychologist about the use of this tool. Treatment receipt, i.e., "the extent to which the patient understands the strategies or techniques taught, and demonstrates the capacity to use them" (Bellg et al., 2004) was verified at different times during the training process (by means of direct questions).

The programme consisted of individual sessions lasting two hours once a week for at least six weeks. The patients performed MVI tasks of increasing difficulty, the content of which was divided into four steps. The rate of progression through these steps was flexible and could be adapted to each patient. Completion of the entire programme required at least six sessions but additional sessions were proposed if necessary to complete the programme at the patient's rhythm of training. Over the duration of the training programme, the role of the neuropsychologist was to teach how to construct visual scenes, and to provide continuous guidance throughout the training sessions.

The screening test. This test was based on 12 size comparisons (De Renzi \& Lucchelli, 1994; e.g., "Which object is larger, a knife blade or a saw blade?"). We used this test to probe basic visual imaging abilities and, therefore, be able to exclude patients presenting with severe MVI impairment (incompatible with the realisation of the facilitation programme). Thus, based on unpublished empirical data obtained in a group of normal subjects, a minimal score of $9 / 12$ was deemed necessary to continue the programme.

The external visualisation exercise. This included 10 verbal items that the patient had to imagine and describe. (We called this step "external" visualisation because the purpose of the exercise was to imagine an object and not the participant him/herself.) The instruction ran as follows: "I will tell you the name of an object (e.g., an onion), imagine it and describe its colour, shape, size, consistency and every detail that you see." Each item description was checked for accuracy using a list of typical traits. For each item, the patient had to describe static aspects (e.g., colour, shape) and an action carried out with the item (e.g., slicing an onion). The action was imagined to be performed either by him/herself or someone else (no instruction was given as to who was supposed to act, since the patient was asked to concentrate on an object having changed in its physical appearance owing to an action).

The construction exercise. This part of the programme consisted of figuring out complex scenes bringing into play several characters. The goal was twofold, i.e., to increase the complexity of the scene by comparison with the previous step and to introduce several characters performing different complex actions. Five items were proposed with, for each one, a first training step and a subsequent scene construction step. During the training phase, a neuropsychologist guided the patient, starting from a general idea (e.g., "A cook prepares a meal"), to more precise subheadings (e.g., "The cook is in front of a metallic table"). Within subheadings, detailed items were distinguished (e.g., "On the table, you can see butter, some onions and mushrooms..."). The present step was considered to be external since the participant was asked to imagine some characters and focus primarily on them, their actions and the context of the scene rather than the patient him/herself. Responses were checked for accuracy in terms of their relation to each subheading, but no list of typical or expected responses was used. The number of headings and sub-headings, as well as details produced by the patients was recorded. For the scene 
construction phase, an item similar to the one used in the training section was proposed (e.g., "Can you imagine the job you would most enjoy and describe it, as we just did with the example of the cook?").

The self-visualisation exercise. This was the training phase that shared the most similarities with AbM since the patients were asked to visualise themselves within a given scenario, to imagine it as though they were actually living the scene and to describe whatever details, sensations or feelings that came to mind. The presentation was similar to that of the previous exercise, comprising a training (e.g., "You are heading towards a hotel reception desk") and a scene construction phase (e.g., "Your room is not ready; picture yourself in the hotel bar"). The difference was that the participant was asked to focus his/her attention on him/herself, i.e., using internal, personal knowledge. As for the previous step, we recorded the number of headings, sub-headings and details produced by the patients.

\section{Statistical analysis}

Percentages of MS patients with deficient scores on the neuropsychological baseline examination were calculated for each test, and are presented in Table 2.

Patients' and controls' scores on the MCT were analysed across the different life periods by means of a repeatedmeasures ANOVA. When appropriate, the ANOVA was followed by Tukey's HSD post-hoc test.

The relationship between AbM performance and, on the one hand, executive function scores, and, on the other hand, the results on anterograde memory tests, were analysed by means of a "frontal lobe function index" (including the PASAT, the phonological and categorical verbal fluency tests) and an "anterograde memory index" (including the SRT/RAVLT, the RMTW and the figure recall test from the AMIPB). For each index, only the patients who had completed the three tests were taken into account for the analysis. Firstly, the performance of each patient was categorised as "preserved" or "deficient" for all these tests. To be classified as "preserved" for the "frontal lobe function index" a patient's score had to be either flawless or to show only one failure. With regard to the "anterograde memory index", "preserved" performance corresponded to at least one score in the normal range on the RMT, plus normal performance on one of the two free recall tests. On this basis, we performed a point-biserial correlation analysis between each index and the MCT global score.

Comparison between MS patients and healthy controls for Personal Semantics (AMI) was performed with the Student $t$-test.

For statistical analysis involving the cognitive facilitation programme results, we calculated the difference between the MCT global score of MS patients and healthy controls in pre- and post-facilitation by means of a repeated-measure ANOVA, followed by Tukey's HSD post-hoc test. The effect size was then estimated with the partial $\eta^{2}$ value. An individual analysis was also conducted for each patient comparing their global score on the MCT before and after administration of the facilitation programme, using the $\chi^{2}$ test (see Levaux, Laroi, Offerlin-Meyer, Danion, \& Van der Linden, 2011 for statistical analyses for individual comparisons pre-/posttreatment). The same procedure for individual level analysis was used for the healthy controls global score on the MCT.

\section{RESULTS}

The percentages of patients who obtained scores under the normal range for the neuropsychological baseline examination are presented in Table 2.

\section{Autobiographical memory assessment}

Concerning the comparison of scores on the MCT per life period between the two groups (MS patients and healthy controls), the ANOVA showed a main effect of group, $F(1,58)=85.011, p<.001$, and a main effect of life period, $F(3,174)=17.834, p<.001$. However, no significant interaction effect was observed, $F(3,174)=$ $1.430, p=.235$. Post-hoc analysis revealed that healthy controls had significantly higher scores for each life period relative to patients $(p<.001$ in every case). Within the patient group, significantly lower scores were obtained for the 0-9 years and the past year life periods in comparison to the 20-to-(current age - 1 year) life period $(p<.001$ in all cases). No other comparisons reached the statistical significance threshold for the MS group. Within the healthy control group, no significant differences between life periods were observed, excepted for the 0-9 years life period for which a lower score was obtained compared to the three remaining life periods ( $p$ $<.001$ for the comparison with the 10-19 years and the 20-to-(patient's current age - 1 year) life periods and $p<$ 
.05 for the comparison with the past year life period).

Correlation analyses addressing the "frontal lobe function index" were carried out on the scores of 20 patients, while the "anterograde memory index" was calculated on the performance of 15 patients. We found a significant positive correlation between the "frontal lobe function index" and the MCT global score $\left(r_{\mathrm{pb}}=0.458, p<.05\right)$. On the contrary, we found no significant correlation between the "anterograde memory index" and the MCT results $\left(r_{\mathrm{ph}}=0.284, p=.304\right)$.

\section{Personal semantics}

Concerning the AMI personal semantics, scores did not differ significantly between MS patients and healthy controls, $t(48)=-.036, p=.970$.

\section{Autobiographical memory facilitation programme}

It can be seen in Figure 1 that the group analyses showed a significant main group effect on the MCT global score, $F(1,18)=21.813, p<.001$, with an important effect size, $\eta^{2}=.55$. Post-hoc analyses revealed poorer scores for the patient group $(p<.001)$. A significant effect of time of assessment was observed, $F(1,18)=$ $108.147, p<.001$, with a marked effect size, $\eta^{2}=.86$. Post-hoc analyses showed a significant effect only for the comparison of the pre- and post-facilitation scores of MS patients $(p<.001)$. A significant interaction effect was also shown between the group and time of assessment factors, $F(1,18)=89.663, p<.001$, with a large size effect, $\eta^{2}=.83$. Post-hoc analysis revealed that the scores achieved by MS patients on the MCT prior to the facilitation programme were significantly lower than that of healthy controls $(p<.001)$ but the post-facilitation assessment showed no significant difference between the two groups $(p=.436)$.

Figure 1. Group analyses of the MCT global score for MS patients pre-and post-facilitation and healthy controls $(* p<.001)$

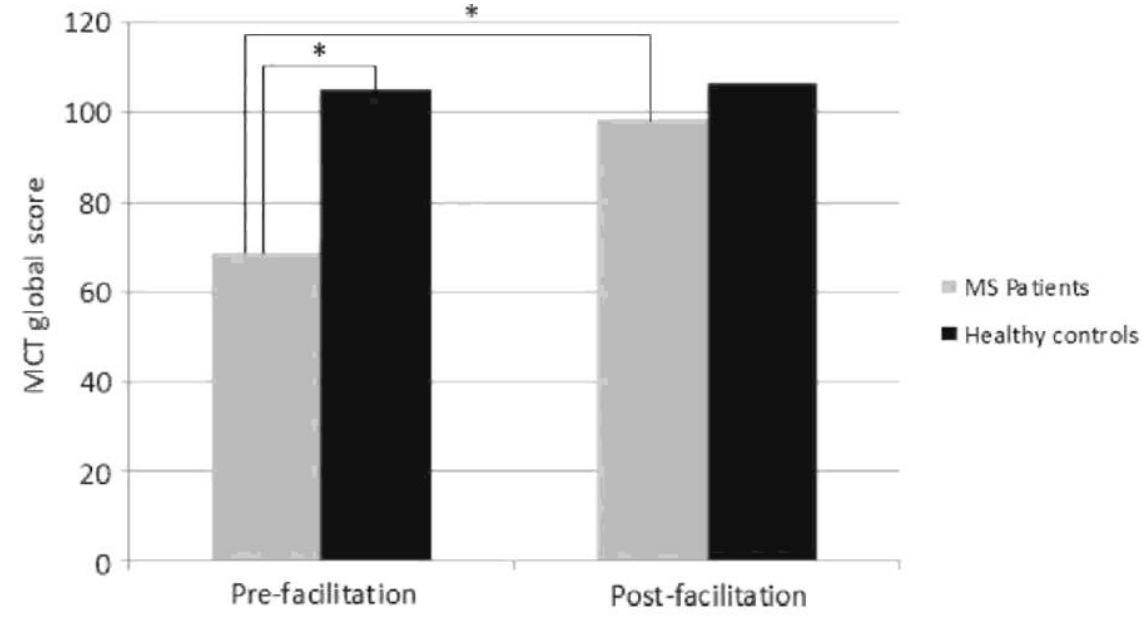

The individual analyses revealed a significant improvement in the MCT global score for all 10 patients after the rehabilitation sessions (see Table 3). Moreover, seven of them showed scores in the normal interval of their MCT global score ( $\geq 74 \%$ of the maximum total score corresponding to a score of 88/120) after MVI training. For the 10 healthy controls, no significant change in MCT global score was observed between the two assessments (Table 3).

Bearing in mind that our primary goal was to improve everyday life functioning in our patients, we also collected, after the facilitation programme, their comments about the perceived benefits on the second MCT testing and in everyday life (see Appendix 1). Globally, all the patients' comments confirmed the positive effects of the cognitive intervention. Among them, the most frequently evoked benefits were greater ease of retrieval, a greater number of details and better self-visualisation. Positive comments were also conveyed by the patients concerning treatment receipt: they mentioned easy and effective use and transfer of this technique in their daily life functioning. 
TABLE 3 Individual analyses of the MCT global score for MS patients pre-and post-facilitation and healthy controls

\begin{tabular}{lcccccc}
\hline & \multicolumn{3}{c}{ MS Patients } \\
\cline { 2 - 7 } & Pre - & Post - & $\chi^{2}$ & Session 1 & Session 2 & $\chi^{2}$ \\
\hline 1 & 40 & 80 & $26.67 * *$ & 103 & 105 & 0.14 \\
2 & 57 & 86 & $14.55 * *$ & 97 & 100 & 0.25 \\
3 & 47 & 93 & $36.27 * *$ & 106 & 104 & 0.15 \\
4 & 66 & 97 & $18.38 * *$ & 116 & 113 & 0.86 \\
5 & 76 & 102 & $14.7 * *$ & 102 & 101 & 0.03 \\
6 & 80 & 117 & $38.79 * *$ & 110 & 112 & 0.24 \\
7 & 87 & 114 & $22.32 * *$ & 105 & 107 & 0.16 \\
8 & 85 & 112 & $20.65 * *$ & 105 & 107 & 0.16 \\
9 & 84 & 101 & $6.82 *$ & 103 & 104 & 0.04 \\
10 & 68 & 87 & $6.58 *$ & 103 & 111 & 2.76 \\
\hline$* p<.01$ and ${ }^{* *} p<.001$. MCT: Modified Crovitz Test; MS: multiple sclerosis
\end{tabular}

\section{DISCUSSION}

In this study, we have documented AbM impairment concomitant with preserved personal semantics in a group of non-clinically depressed RRMS patients with mild-to-moderate disease severity. One of the interesting findings of the present study, concerning cognitive functioning in MS, is that MS patients, regardless of their scores on different cognitive functions, presented AbM impairment. We would like to suggest that this result is of interest since it reveals, for the first time to our knowledge, that subtle cognitive impairment in MS patients may affect only AbM performance. Moreover, despite the fact that no cognitive profile can be drawn owing to the heterogeneous nature of lesions inherent in this condition, AbM seems to be particularly sensitive to MS neuropathology, which argues for its routine assessment. Our findings are contrary to those obtained by Paul et al. (1997) but in accord with those of Kenealy et al. (2002). However, both Paul et al. and Kenealy et al. used the Autobiographical Memory Interview (AMI; Kopelman et al., 1989). While the personal semantics section in the AMI is very useful and has a good sensitivity, its episodic component is recognised as a short measure of autobiographical assessment, with imprecise temporal resolution and a rather poor sensitivity (McKinnon, Black, Miller, Moscovitch, \& Levine, 2006; Piolino, Desgranges, Benali, \& Eustache, 2002). Moreover, in those two studies the MS subtypes were not controlled, and the patient populations differed widely in terms of disease severity. By contrast, the use of a more stringent AbM test as well as the selection of a specific subtype of MS patients, whose disease severity was also controlled, could be considered as obvious advantages of the present study, which furthermore sets off the examination of processes that could potentially underlie AbM deficit in MS. Indeed, we investigated the links between AbM, executive functions and anterograde memory patterns of performance in order to confirm or otherwise potential interactions, to try and gain a better understanding of $\mathrm{AbM}$ deficit characteristics. We have shown an association between the AbM scores and executive functions but none with anterograde memory performance. This result suggests that AbM deficits could be due to retrieval difficulties provoked by frontal lobe dysfunction, above and beyond medial temporal lobe dysfunction. This finding is in accord with several studies carried out in various clinical conditions involving the frontal lobe and accounting for AbM impairment in terms of defective retrieval strategies (Delia Sala, Laiacona, Spinnler, \& Trivelli, 1993; Kopelman, Stanhope, \& Kingsley, 1999; Matuszewski et al., 2006; Zarei et al., 2010).

Although AbM impairment in our MS patients covered the whole lifespan, within-group comparisons showed a reversed U-shape profile of scores across the life periods. This pattern of results is very likely due to the higher recall rate of memories from the early adulthood period relative to the other life periods, i.e., the reminiscence bump phenomenon. Privileged access to memories belonging in the reminiscence bump period has been frequently suggested in the literature (see Conway \& Rubin, 1993, for a review). We would like to suggest that, although the AbM scores before the facilitation programme were deficient over lifespan, the higher scores observed for the 20-to-(current age - 1 year) life period could be interpreted as a relative preservation of the reminiscence bump. More to the point, this result suggests that easier access to memories from the reminiscence bump reduces the reliance on retrieval strategies.

In the same vein, the effective outcome of our MVI-based facilitation programme corroborates, to a greater degree, the hypothesis of retrieval strategy deficits related to prefrontal cortex dysfunction. Indeed, the MVI training effects would have been different had the encoding or consolidation processes been defective. This is why our programme is best characterised as a "facilitation programme" (Seron \& Van der Linden, 2000) as it 
uses a critical AbM component in an intensified and optimised way. In other words, the facilitation programme may have triggered the use of and reliance on the cueing role of MVI (Huijbers et al., 2011; Rubin, 2005) during both memory retrieval and search for details concerning a unique past event.

The positive outcome does not seem due to a learning effect on the MCT since no changes were observed in the control group's performance. However, we acknowledge the fact that healthy controls had little margin to improve their scores on the second assessment. More compelling evidence can be found in a study involving epileptic patients using the MCT (Voltzenlogel, Després, Vignal, Kehrli, \& Manning, 2007), which showed that scores remained unchanged between the two assessment sessions carried out in the study. Moreover, we are currently studying a group of MS patients using a different test, the Autobiographical Interview (Al; Levine, Svoboda, Hay, Winocur, \& Moscovitch, 2002; kindly communicated by the author, Brian Levine to one of us, LM.). The first results of our on-going study, in which the AI is taken twice with no intervention between the two sessions, show that AbM scores remain stable (Ernst et al., 2012). Taken together, these results seem to indicate that $\mathrm{AbM}$ performance, even using different tests, does not seem prone to learning effects.

It is important to note that significantly improved performance on the post-MVI programme MCT was observed in every case, and normalised scores were verified for nearly all patients. We turn now to the important issue of benefit transfer in daily life. This being a central concern in neuropsychological rehabilitation, several studies have dealt with this topic, but failed to show such a transfer (Glisky \& Glisky, 2002; Johnstone \& Stonnington, 2009). It is therefore of significance that in the present study, we found that improved performance on the AbM test was accompanied by the patients' reports acknowledging easier retrieval of personal memories, a greater number of details and a feeling of being able to "relive the event", not only during the post-facilitation AbM assessment, but also in everyday life. According to our patients' comments, it seems that they were able to integrate and "automate" the use of MVI as a tool in their daily life. Additionally, spontaneous comments by some patients' relatives further supported the notion of effective transfer of this technique into everyday life. More precisely, they observed changes in the evocation and sharing of memories with the patients, in particular concerning the quantity of details.

Several factors could explain why the transfer of benefits from the facilitation programme operated efficiently in the present study. Firstly, our facilitation programme targeted AbM, which is, in essence, memory of real-life events. Secondly, the patients were taught throughout the facilitation training, how to use a specific strategy, i.e., a mental process that could be applied to real-life situations, and which was abundantly explained to the patients during the facilitation sessions. Relating to the latter point, the use of compensatory strategies has been described as more efficient in terms of transfer in everyday life, especially concerning memory rehabilitation, than the use of training methods which aim at restoring a damaged function (Glisky \& Glisky, 2002). Finally, according to the study of Evans, Wilson, Needham, and Brentnall (2003), the main predictors of the good use of compensation strategies in everyday life are: younger age, mild severity of impairment, deficit specificity, and premorbid use of strategies. In the present study, this facilitation programme was proposed to relatively young patients with a selective AbM deficit. Moreover, the strategy we taught must have been spontaneously used before the illness since it is based on a critical component necessarily required for AbM. From a neuroanatomical point of view, although we yet have to obtain neuroanatomical data, we would like to suggest that emphasis put on the role of MVI in AbM and, by the same token, on the posterior cerebral regions (Hassabis et al., 2007), could have compensated for the weakness of the retrieval strategy processes supported by the prefrontal cortex (Botzung et al., 2008; Manenti et al., 2010), which is more affected in MS in comparison to posterior brain regions (Arnett, 2003; Bendfeldt et al., 2009; Benedict et al., 2002; Sperling et al., 2001).

One of the limitations of the present work is due above all to the small size of the group that underwent the facilitation programme. However, despite the latter, we obtained statistical evidence of AbM improvement, which indicates the clinical interest of the study. To overcome the above-mentioned limitation and to confirm our hypotheses concerning the initial retrieval strategy deficit, a neuroimaging study involving a larger group of MS patients has been initiated (for preliminary results, see Ernst et al., 2012). A further limitation of the work concerns the fact that the post-facilitation AbM assessment did not follow a blind-investigator study design and, for the time being, the presence of a "nursing effect" cannot be altogether ruled out. Finally, long-term follow-up measures are required to examine the robustness of the treatment effects.

On the positive side, the present work is, to the best of our knowledge, the first study focusing on AbM cognitive rehabilitation in MS, and our findings seem to be promising and encourage future investigations. Moreover, the development of this kind of intervention appears as a priority considering the absence, or at least the suboptimal effectiveness, of current pharmacological treatments for cognitive impairment in MS (Krupp et al., 2011; Lovera et al., 2010; Thompson, Toosy, \& Ciccarelli, 2010), especially considering the well-known deleterious impact of 
Published in : Neuropsychological Rehabilitation (2012)

Status : Postprint (Author's version)

cognitive deficits on MS patients' everyday lives.

\section{REFERENCES}

Addis, D. R., Sacchetti, D. C., Ally, B. A., Budson, A. E., \& Schacter, D. L. (2009). Episodic simulation of future events is impaired in mild Alzheimer's disease. Neuropsychologia, 47, 2660-2671.

Allen, D. D., Goldstein, G., Heyman, R. A., \& Rondinelli, T. (1998). Teaching memory strategies to persons with multiple sclerosis. Journal of Rehabilitation Research and Development, 35, 405-410.

Arnett, P. A. (2003). Neuropsychological presentation and treatment of demyelinating disorders. In P. W. Halligan, U. Kischka, \& J. C. Marshall (Eds.), Handbook of clinical neuropsychology (pp. 368-386). Oxford, UK: Oxford University Press.

Basso, M. R., Lowery, N., Ghormley, C., Combs, D., \& Johnson, J. (2006). Self-generated learning in people with multiple sclerosis. Journal of the International Neuropsychological Society, 12, 640-648.

Beck, A. T., \& Steer, R. A. (1993). Beck depression inventory manual. San Antonio, TX: The Psychological Corporation.

Bellg, A. J., Borrelli, B., Resnick, B., Hecht, J., Minicucci, D. S., Ory, M., ... Treatment Fidelity Workgroup of the NIH Behavior Change Consortium. (2004). Enhancing treatment

fidelity in health behavior change studies: Best practices and recommendations from the NIH Behavior Change Consortium. Health Psychology, 23, 443-451.

Bendfeldt, K., Kuster, P., Traud, S., Egger, H., Winklhofer, S., Mueller-Lenke, N.,... Borgwardt, S. J. (2009). Association of regional gray matter volume loss and progression of white matter lesions in multiple sclerosis - A longitudinal voxel-based morphometry study. NeuroImage, 45, 60-67.

Benedict, R. H. B., Bakshi, R., Simon, J. H., Priore, R., Miller, C., \& Munschauer, F. (2002). Frontal cortex atrophy predicts cognitive impairment in multiple sclerosis. Journal of Neuropsychiatry and Clinical Neurosciences, 14, 44-51.

Botzung, A., Denkova, E., Ciuciu, P., Scheiber, C, \& Manning, L. (2008). The neural bases of the constructive nature of autobiographical memories studied with a self-paced fMRI design. Memory, 16, 351-363.

Buschke, H., \& Fuld, P. A. (1974). Evaluating storage, retention and retrieval in disordered memory and learning. Neurology, 24, $1019-1025$.

Calabrese, P. (2006). Neuropsychology of multiple sclerosis: An overview. Journal of Neurology, 253, 10-15

Chiaravalloti, N. D., \& DeLuca, J. (2002). Self-generation as a means of maximizing learning in multiple sclerosis: An application of the generation effect. Archives of Physical Medicine Rehabilitation, 83, 1070-1079.

Chiaravalloti, N. D., DeLuca, J., Moore, N. B., \& Ricker, J. H. (2005). Treating learning impairments improves memory performance in multiple sclerosis: A randomized clinical trial. Multiple Sclerosis, 11, 58-68.

Chiaravalloti, N. D., Demaree, H., Gaudino, E., \& DeLuca, J. (2003). Can the repetition effect maximize learning in multiple sclerosis? Clinical Rehabilitation, 17, 58-68.

Conway, M. A., \& Rubin, D. C. (1993). The structure of autobiographical memory. In A. E. Collins, S. E. Gathercole, M. A. Conway, \& P. E.M. Morris (Eds.), Theories of memory (pp. 103-137). Hove, Sussex, UK: Lawrence Erlbaum Associates.

Coughlan, A., \& Hollows, S. (1985). The adult memory and information processing battery. Saint James Hospital, Leeds.

Delia Sala, S., Laiacona, M., Spinnler, H., \& Trivelli, C. (1993). Autobiographical recollection and frontal damage. Neuropsychologia, 31 823-839.

De Renzi, E., \& Lucchelli, F. (1994). Are semantic systems separately represented in the brain? The case of living category impairment Cortex, 30, 3-25.

Drew, M., Tippett, L. J., Starkey, N. J., \& Isler, R. B. (2008). Executive dysfunction and cognitive impairment in a large community-based sample with multiple sclerosis from New Zealand: A descriptive study. Archives of Clinical Neuropsychology, 23, 1-19.

Ernst, A., Botzung, A., Voltzenlogel, V., Blanc, F., De Seze, J., Sellal, F., \& Manning, L. (2012). Autobiographical memory facilitation in multiple sclerosis: A neuropsychological and neuroimaging approach. Brain Impairment, 13, 143.

Evans, J. J., Wilson, B. A., Needham, P., \& Brentnall, S. (2003). Who makes good use of memory-aids? Results of a survey of 100 people with acquired brain injury. Journal of the International Neuropsychological Society, 9, 925-935. 
Glisky, E. L., \& Glisky, M. L. (2002). Learning and memory impairments. In J.P. Eslinger (Ed.), Neuropsychological Interventions. Clinical Research and Practice (pp. 137-162). New York: The Guilford Press.

Goverover, Y., Hillary, F. J., Chiaravalloti, N. D., Arango-Lasprilla, J. C., \& DeLuca, J. (2009). A functional application of the spacing effect to improve learning and memory in persons with multiple sclerosis. Journal of Clinical and Experimental Neuropsychology, 31, 513522 .

Graham, K., \& Hodges, J. R. (1997). Differentiating the roles of the hippocampal complex and the neocortex in LTM storage: Evidence from the study of semantic dementia and Alzheimer's disease. Neuropsychology, 11, 77-89.

Greenberg, D. L., Eacott, M. J., Brechin, D., \& Rubin, D. C. (2005). Visual memory loss and autobiographical amnesia: A case study. Neuropsychologia, 43, 1493-1502.

Greenberg, D. L., \& Rubin, D. C. (2003). The neuropsychology of autobiographical memory. Cortex, 39, 687-728.

Gronwall, D. M. A. (1977). Paced auditory serial-addition task: A measure of recovery from concussion. Perceptual and Motor Skills, 44, 367-373.

Hamilton, M. (1959). The assessment of anxiety states by rating. British Journal of Medical Psychology, 32, 50-55

Hassabis, D., Kumaran, D., \& Maguire, E. A. (2007). Using imagination to understand the neural basis of episodic memory. Journal of Neuroscience, 27, 14365-14374.

Hildebrant, H., Lanz, M., Hahn, H. K., Hoffmann, E., Schwarze, B., Schwendemann, G., \& Kraus, J. A. (2007). Cognitive training in MS Effects and relation to brain atrophy. Restorative Neurology and Neuroscience, 25, 33-43.

Huijbers, W., Pennartz, C. M. A., Rubin, D. C., \& Daselaar, S. M. (2011). Imagery and retrieval of auditory and visual information: Neural correlates of successful and unsuccessful performance. Neuropsychologia, 49, 1730-1740.

Johnstone, B., \& Stonnington, H. H. (2009). Rehabilitation of neuropsychological disorders. A practical guide for rehabilitation professionals (2nd ed., pp. 1-23). Hove, UK: Psychology Press, Taylor and Francis Group.

Kenealy, P., Beaumont, G., Lintern, T., \& Murrell, R. (2002). Autobiographical memory in advanced multiple sclerosis: Assessment of episodic and personal semantic memory across three time spans. Journal of Neuropsychological International Society, 8, 855-860.

Kopelman, M., Stanhope, N., \& Kingsley, D. (1999). Retrograde amnesia in patients with diencephalic, temporal lobe or frontal lesions Neuropsychologia, 37, 939-958.

Kopelman, M., Wilson, B., \& Baddeley, A. (1989). The Autobiographical Memory Interview: A new assessment of autobiographical and personal semantic memory in amnesic patients. Journal of Experimental and Clinical Neuropsychology, 11, 724-744.

Kremin, H. (2002). L'acces au lexique en denomination d'images: Problemes actuels. Revue Francaise de Psychologie, 47, 77-91.

Krupp, L. B., Christodoulou, C., Melville, P., Scherl, W. F., Pai, L. Y., Muenz, L.R.,... Wishart, H. (2011). Multicenter randomized clinical trial of donepezil for memory impairment in multiple sclerosis. Neurology, 26, 1500-1507.

Kurtzke, J. F. (1983). Rating neurological impairment in multiple sclerosis: An Expanded Disability Status Scale (EDSS). Neurology, 33, $1444-1452$

Levaux, M. N., Lar0i, F., Offerlin-Meyer, I., Danion, J. M., \& Van der Linden, M. (2011). The effectiveness of the attention training technique in reducing intrusive thoughts in schizophrenia: A case study. Clinical Case Studies, 10, 466-484.

Levine, B., Svoboda, E., Hay, J. F., Winocur, G., \& Moscovitch, M. (2002). Aging and autobiographical memory: Dissociating episodic from semantic retrieval. Psychology and Aging, 17, 677-689.

Lovera, J. F., Frohman, E., Brown, T. R., Bandari, D., Nguyen, L., Yadav, V., ... Bourdette, D. (2010). Memantine for cognitive impairment in multiple sclerosis: A randomized placebo-controlled trial. Multiple Sclerosis, 16, 715-723.

Manenti, R., Cotelli, M., Calabria, M., Maioli, C., \& Minussi, C. (2010). The role of retrieval from long term memory depends on strategies A repetitive transcranial magnetic stimulation study. Neuroscience, 166, 501-507.

Manning, L. (2002). Focal retrograde amnesia documented with matching anterograde and retrograde procedures. Neuropsychologia, 40, 28 38.

Matuszewski, V., Piolino, P., de la Sayette, V., Lalevee, C., Pelerin, A., \& Dupuy, B. (2006). Retrieval mechanisms for autobiographical memories: Insights from the frontal variant of frontotemporal dementia. Neuropsychologia, 44, 2386-2397.

McDonald, W. I., Compston, A., Edan, G., Goodkin, D., Hartung, H. P., Lublin, F.D., ... Wolinsky, J. S. (2001). Recommended diagnostic criteria for multiple sclerosis: Guidelines from the International Panel on the diagnosis of multiple sclerosis. Annals of Neurology, 50, 121- 
Published in : Neuropsychological Rehabilitation (2012)

Status : Postprint (Author's version)

127.

McKinnon, M. C., Black, S. E., Miller, B., Moscovitch, M., \& Levine, B. (2006). Autobiographical memory in semantic dementia: Implications for theories of limbic-neocortical interaction in remote memory. Neuropsychologic 44, 2421-2429.

Mendozzi, L., Pugnetti, L., Motta, A., Barbieri, E., Gambini, A., \& Cazullo, C. L. (1998). Computer-assisted memory retraining of patients with multiple sclerosis. Italian Journal of Neurological Sciences, 19, S431-S438.

Miller, L. A., Caine, D., Harding, A., Thompson, E. J., Large, M., \& Watson, J. D. G. (2001). Right medial thalamic lesion causes isolated retrograde amnesia. Neuropsychologia, 39, 1037-1046.

Montoya, A., Pelletier, M., Menear, M., Duplessis, E., Richer, F., \& Lepage, M. (2006). Episodic memory impairment in Huntington's disease: A meta-analysis. Neuropsychologia, 44, 1984-1994.

Moscovitch, M., Rosenbaum, R. S., Gilboa, A., Addis, D. R., Westmacott, R., Grady, C., ... Nadel, L. (2005). Functional neuroanatomy of remote episodic, semantic and spatial memory: A unified account based on multiple trace theory. Journal of Anatomy, 207, 35-66.

Moulton, S. T., \& Kosslyn, S. M. (2009). Imagining predictions: Mental imagery as mental emulation. Philosophical Transactions of the Royal Society Biological Sciences, 364, 1273-1280.

O'Brien, A. R., Chiaravalloti, N., Goverover, Y., \& DeLuca, J. (2008). Evidenced-based cognitive rehabilitation for persons with multiple sclerosis: A review of the literature. Archives of Physical Medicine and Rehabiliation, 89, 761-769.

Paul, R., Blanco, C., Hames, K., \& Beatty, W. (1997). Autobiographical memory in multiple sclerosis. Journal of Neuropsychological International Society, 3, 246-251.

Piolino, P., Desgranges, B., Benali, K., \& Eustache, F. (2002). Episodic and semantic remote autobiographical memory in ageing. Memory, 10, 239-257

Rao, S. M., Leo, G. J., Bernardin, L., \& Unverzagt, F. (1991). Cognitive dysfunction in multiple sclerosis: I. Frequency, patterns, and prediction. Neurology, 41, 685-691.

Rapp, B., \& Camarazza, A. (1993). On the distinction between deficits of access and deficits of storage: A question of theory. Cognitive Neuropsychology, 10, 113-141.

Rasmussen, A. S., \& Habermas, T. (2011). Factor structure of overall autobiographical memory usage: The directive, self and social functions revisited. Memory, 19, 597-605.

Rey, A. (1964). L'Examen clinique en Neuropsychologic Paris: PUF

Rogers, J. M., \& Panegyres, P. K. (2007). Cognitive impairment in multiple sclerosis: Evidence-based analysis and recommendations Journal of Clinical Neuroscience, 14, 919-927.

Rubin, D. C. (2005). A basic-systems approach to autobiographical memory. Current Directions in Psychological Science, 14, 79-83.

Rubin, D. C., \& Greenberg, D. L. (1998). Visual memory-deficit amnesia: A distinct amnesia presentation and etiology. Proceedings of the National Academy of Sciences, USA, 95, 5413-5416.

Santiago, O., Guardia, J., Casado, V., Carmona, O., \& Arbizu, T. (2007). Specificity of frontal dysfunction in relapsing-remitting multiple sclerosis. Archives of Clinical Neuropsychology, 22, 623-629.

Sartori, E., \& Edan, G. (2006). Assessment of cognitive dysfunction in multiple sclerosis. Journal of the Neurological Sciences, 245, 169175 .

Seron, X., \& Van Der Linden, M. (2000). Objectifs et strategies de la revalidation neuropsychologique. In M. Van Der Linden \& M. Van Der Linden (Eds.), Traité de Neuropsychologie Clinique. Marseille: Solal; Tome II.

Smith, S., Souchay, C., \& Conway, M. (2009). Overgeneral autobiographical memory in Parkinson's disease. Cortex, 27, $987-1003$.

Solari, A., Motta, A., Mendozzi, L., Pucci, E., Forni, M., Mancardi, G., \& Pozzilli, C. (2004). Computer-aided retraining of memory and attention in people with multiple sclerosis: A randomized, double-blind controlled trial. Journal of Neurological Sciences, 222, 99-104.

Sperling, R. A., Guttmann, C. R. G., Hohol, M. J., Warfield, S. K., Jakab, M., Parente, M., ... Weiner, H. L. (2001). Regional magnetic resonance imaging lesion burden and cognitive function in multiple sclerosis. A longitudinal study. Archives of Neurology, 58, 115-121.

Suddendorf, T., \& Corballis, M. C. (1997). Mental time travel and the evolution of the human mind. Genetic, Social, and General Psychology Monographs, 123, 133-167. 
Svoboda, E., McKinnon, M. C, \& Levine, B. (2006). The functionnal neuroanatomy of autobiographical memory: A meta-analysis. Neuropsychologia, 44, 2189-2208.

Thompson, A. J., Toosy, A. T., \& Ciccarelli, O. (2010). Pharmacological management of symptoms in multiple sclerosis: Current approaches and future directions. Lancet Neurology, 9, 1182-1199.

Trapp, B. D., \& Nave, K. M. (2008). Multiple sclerosis: An immune or a neurodegenerative disorder?. Annual Review of Neuroscience, 31, 247-269.

Tulving, E. (1985). Memory and consciousness. Canadian Psychology, 26, 1-12.

Tulving, E., Schacter, D. L., McLachlan, D. R., \& Moscovitch, M. (1988). Priming of semantic autobiographical knowledge: A case study of retrograde amnesia. Brain and Cognition, 8, 3-20.

Voltzenlogel, V., Despres, O., Vignal, J. P., Kehrli, P., \& Manning, L. (2007). One-year postoperative autobiographical memory following unilateral temporal lobectomy for control of intractable epilepsy. Epilepsia, 48, 605-608.

Voltzenlogel, V., Despres, O., Vignal, J. P., Steinhoff, B. J., Kehrli, P., \& Manning, L. (2006). Remote memory in temporal lobe epilepsy. Epilepsia, 47, 1329-1336.

Warrington, E. (1984). Recognition Memory Test. Windsor: NFER-Nelson.

Warrington, E., \& Shallice, T. (1979). Semantic access dyslexia. Brain, 102, 43-63.

Warrington, E., \& James, M. (1991). The Visual Object and Space Perception Battery (VOSP). Suffolk UK: Thames Valley Test Company.

Warrington, E., James, M., \& Maciejewski, C. (1986). The WAIS as a lateralizing and localizing instrument: A study of 656 patients with unilateral cerebral lesions. Neuropsychologia, 24, 223-239.

Wechsler, D. (1989). Echelle d'intelligence de Wechsler pour adultes forme révisée WAIS-R. Paris: Edition du centre de Psychologie Appliquée.

Wilson, B. A. (1987). Rehabilitation of memory. New York, London: The Guilford Press

Wilson, B. A. (2008). Memory rehabilitation. Integrating theory and practice. New York, London: The Guilford Press.

Zakzanis, K K. (2000). Distinct neurocognitive profiles in multiple sclerosis subtypes. Archives of Clinical Neuropsychology, 15, 115-136.

Zarei, M., Pourtemad, H. R., Bak, T., \& Hodges, J. R. (2010). Autobiographical memory in progressive supranuclear palsy. European Journal of Neurology, 17, 238-241.

\section{APPENDIX 1}

GG: "Last time it was more elusive. I could come up with more details, today, if I had the very same words, I'd tell much more things. My feeling is that there's a difference, concerning the details, above all, but also in terms of giving more meanings to words; I've found that. All in all, it's there, more easily. My people are aware of it. I happen to talk about things that I wouldn't before. It often happens with my parents as my mother talks about me when I was a kid and I didn't remember those things, but now I do."

MH: "I'm much more aware of details now... Any recollection I remember, opens up, so to say, several other memories with you and at home as well."

SR: "It was easier this time. The first time I found it really hard; it's the same exercise, though. Last time I couldn't find things in my memory. Today, they just pop out. I realise I can look for in my memory, a word I mean, I'm able to look for it. I say it'll be there and it's there. I have the feeling that I don't need to look for it as much as before. It's not mixed up in my head, I have a thing, I deal with that and little by little things come out. Before the sessions, I used to feel paralysed and nothing would come out. Lots of small recollections are there now. Something appears and other things arrive on the top of that and it grows up."

MS: "I feel it's different now, there is this idea of being in the context. I'm really re-living the thing. I remember the first session and no, it's not the same thing. I carry out the exercise more carefully. I re-live the scene; I'm able to see I'm in. I'm good at re-visualising the situation. Before the sessions, I was not very good; it was much more difficult to remember; now recollections arrive easily. I really realise how different it is. I can see that in my everyday life as well." 